Please do not remove this page

RMIT

UNIVERSITY

\title{
Geometric model generation for CFD simulation of blood and air flows
}

Ding, Songlin; Tu, Jiyuan; Cheung, Chi Pok

https://researchrepository.rmit.edu.au/esploro/outputs/9921863304501341/filesAndLinks?institution=61RMIT_INST\&index=null

Ding, S., Tu, J., \& Cheung, C. P. (2007). Geometric model generation for CFD simulation of blood and air flows. Proceedings of the 1st International Conference on Bioinformatics and Biomedical Engineering, 1335-1338. https://doi.org/10.1109/ICBBE.2007.345

Published Version: https://doi.org/10.1109/ICBBE.2007.345

Repository homepage: https://researchrepository.rmit.edu.au

(c) 2007 IEEE. Personal use of this material is permitted. However, permission to reprint/republish this material for advertising or promotional purposes or for creating new collective works for resale or redistribution to servers or lists, or to reuse any copyrighted component of this work in other works must be obtained from the IEEE.

Downloaded On 2023/04/26 17:42:18 +1000 


\section{Geometric Model Generation for CFD Simulation of Blood and Air Flows}

\author{
S. Ding, J. Tu, C.P. Cheung \\ School of Aerospace, Mechanical and \\ Manufacturing Engineering \\ RMIT University \\ Victroria, Australia \\ \{songlin.ding, jiyuan.tu, \\ chipok.cheung\}@rmit.edu.au
}

\author{
R. Beare, T. Phan, D. Reutens \\ Monash Medical Center \\ Monash University \\ Victorial, Australia \\ \{richard.bear, Thanh.Phan, \\ david.reutens\}@med.monash.edu.au
}

\author{
Frank Thien \\ Alfred Hospital and Monash University \\ Victorial, Australia \\ frank.thien@med.monash.edu.au
}

\begin{abstract}
A new adaptive algorithm is developed for the reconstruction of geometric models of carotid arteries and human airways from CT images. Based on the patient-specific geometric models, Computational Fluid Dynamics (CFD) models of patient's blood and air flows are constructed to calculate hemodynamic parameters and particle deposition patterns for patient-specific clinical applications.
\end{abstract}

Keywords- Geometric Modeling, CFD, Vascular Disease, Respiratory Disease

\section{INTRODUCTION}

Computational Fluid Dynamics (CFD) simulation of blood and air flow has been a subject of significant research effort. Over the last decade great progress has been made in computational modelling of blood flows to measure hemodynamic variables $[1,2]$. There have also been numerous studies of aerosol deposition in the respiratory system [3]. However the application of these techniques to clinical evaluation of individual patients has been limited because it is very difficult to generate the accurate anatomic geometric models upon which the approaches depend. The theoretical and experimental studies are performed based on simplified or idealized models which are inconsistent with real situations. Inaccurate models not only affect the accuracy of CFD calculation of particle flows, but may also lead to incorrect conclusions which could be very dangerous in clinical practice.

Many researches [4,5] have been performed in the reconstruction of geometric models and some commercial software packages are available as well. However, there are many problems in the geometries generated by these packages. The models can not be utilized in CFD study directly. Extensive work has to be done on the modification of these models to meet the CFD requirement, which is extremely timeconsuming. [6]. This hinders the downstream research about air and blood flows in which large amount of tests should be run on different models, and is the bottle neck for the clinical application of CFD results. There is a pressing need to develop a robust, automatic method to generate the patient-specific geometric models for downstream CFD and clinical applications.

This paper presents an improved algorithm to automatically generate geometric models of human airways and carotid vessels from CT (Computed Tomography) and MR (Magnetic Resonance) images for clinical applications. Based on these models, patient specific modeling of blood flow and air flow is carried out. Optimal therapeutic decisions and strategies could be adopted according to the patient-specific engineering information generated from the simulation processes

\section{Generation OF GeOMEtric Models}

\section{A. Source Data}

The geometric models are generated from medical image data, such as CT or MRI. The first step in the process of model creation is the generation of a mask that defines the structure of interest. This process is known as segmentation and can be carried out manually by an operator. Region growing methods, in which a seed region inside the structure of interest is grown by iteratively adding border voxels that satisfy an inclusion criteria, are examples of automated methods traditional used to segment complex structures. However, leakage, which occurs when a growing region expands into the background outside the structure of interest, is a common problem when applying region growing to low contrast or noisy images. This problem has been addressed in the context of airway segmentation by adapting the region growing process to detect leaks as they occur and adjust control parameters accordingly. The leak detection step relies on a model of anticipated airway structure.

An alternative approach presented here uses a well established technique from the field of mathematical morphology called the watershed transform. The watershed transform models a landscape being flooded by rain. Rain falling on the surface of a terrain will flow towards local minima and all water flowing towards the same local minimum is said to have fallen in the same catchment basin. The borders between adjacent catchment basins are known as watershed lines. When applied to image segmentation the image is the terrain, with brightness corresponding to altitude and each 
catchment basin represents a segmented region. Watershed lines correspond to bright regions of the image, so it is common to apply the watershed transform to an edge detected version of the image.
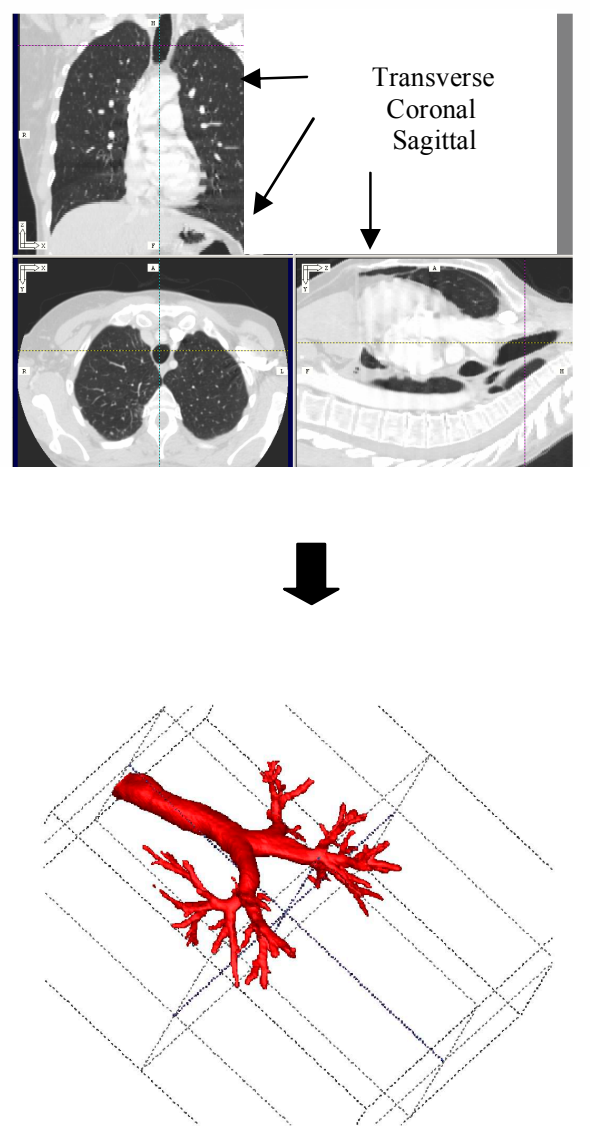

Figure 1. Image processing for airway trees

The presence of noise, texture and other image complexity means that there are generally many more catchment basins than regions of interest - a problem commonly called over segmentation. This problem may be addressed by preprocessing the image in various ways, post processing the segmented regions by merging according to some criterion or a mixture of both. A simpler but very useful alternative used in this work is the marker based watershed described by Meyer and Beucher [7]. The marker based approach imposes minima on the image and removes all others, allowing the number of regions and points within the regions to be defined. The markers can be generated by automated techniques, an operator or a mixture of both and are represented as a set of regions in a marker image. The points in the markers are usually assigned the lowest value for the image type, say 0 , and then used to construct a new relief by recursive conditional erosion [8]:

$$
h_{n+1}=\max \left\{f, a h_{n}\right\}
$$

where $\mathrm{h}_{0}(\mathrm{x})$ the function, with value 0 at the markers and 1 otherwise and $\infty$ denotes the erosion operation. $h \infty$ is a new relief with regional minima corresponding to the markers and the watershed is applied to the new relief.

The lung segmentation was carried out using a markers placed by an operator - an airway marker was placed in the trachea and several background markers were placed on the lung borders. The watershed transform was then applied to the morphological gradient of the CT scan to produce the segmentation shown in Figure 1.

The carotid segmentation process was similar, with an operator marking several points along the carotid. These points were connected together automatically using a minimal path approach and the resulting line was as a marker for the carotid. Background markers were then constructed using knowledge of typical carotid diameter and simple thresholding techniques. The marker based watershed transform was then applied to the gradient of the MR scan.

The critical aspects of applying marker based watershed to practical problems are:

- A different marker is necessary for each region of interest.

- There must be at least 2 markers per image. A single marker will form a catchment basin for the entire image and therefore won't segment a structure of interest.

- Placement of markers is not critical. However a structure marker must lie entirely within the structure. A marker that crosses an image border between region will introduce a path between those regions.

\section{B. Geometric Models}

Once the data point clouds are obtained from above mentioned phase, it is not difficult to construct the triangle model of the geometries in STL format. However, these models can not be applied by CFD packages for the generation of the required geometric mesh. As shown in Figure 2 one more process is needed to convert the triangle model into surface or solid model to facilitate CFD simulations.

Owning to the similarity between the modelling of a mechanical part in CAD/CAM (Computer Aided Design/Manufacturing) systems and the modelling of a human organ in biomedical engineering, both of which are based on the parameters of free-form or sculptured surfaces, B-Spline surfaces modelling used in Reverse Engineering (RE) is applied here to approximate the data clouds obtained from above mentioned segmentation processes. The general procedure includes three steps:

1. Construct initial triangle meshes,

2. Re-parameterise triangle mesh over a quadrilateral domain,

3. Generate B-spline patches

The shortest distance algorithm, which has been used by Eric and Hoppe [9] in surface re-construction, is applied in Step 3 to find the control points in the surface generation 
process, i.e., through minimizing the distance function:

$$
E_{\text {dist }}(S)=\sum_{i=1}^{N} d^{2}\left(P_{i}, S\right)
$$

where: $E_{\text {dist }}($ ) is the distance function, $d$ ( ) is the distance, $P_{i}$ is the point, $S$ is the surface patch..

Some approximation errors are found in the models generated and manual interactions are required before they can be loaded by CFD packages. These errors in geometric modeling can be caused by two factors: (1) lack of sufficient data generated from medical images due to the limitation of CT and MR resolutions and (2) insufficient number of free-form surface patches used in the re-construction of geometric models from source data generated in the image processing phase.

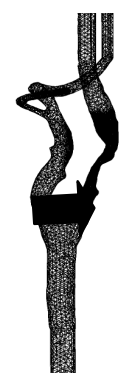

(a)

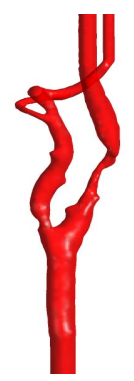

(b)
Figure 2. Geometric model of carotid arteries. (a) Triangle model of carotid artery. (b) Surface model of carotid artery.

Theoretically there are no complete solutions except by employing more advanced scanning machines for the former. For errors caused by the latter, an intelligent "adaptive tolerance" strategy is applied. The general procedure in RE is to assign an overall tolerance for the approximation of the entire models. Owing to the specialty of vessels and airways, if a large uniform tolerance is applied, errors will occur at places with fine structures; if a very small overall tolerance is used, plenty of redundant surface patches will be generated in the main branches. The main idea of the algorithm is to approximate the model with "adaptive tolerances" at various sections. Larger tolerance is used in the main branches of the vessels or airways while a lower tolerance is used at the end of the vessel or after the fifth or sixth generation of the airway where very fine structures exist. Lower tolerance leads to more surface patches $h$. With this strategy, although the tolerance is higher at the smaller bronchus, the overall number of surface patches will not increase significantly and accurate description of the geometries is obtained.

\section{SimUlation OF BloOd AND Air Flows}

Due to the complex geometry of the anatomically real blood vessel and human airways, a commercial CFD code, FLUENT, was utilized to predict the continuum phase flow under both steady and unsteady-state conditions through solutions of the conservation equations of mass and momentum. A pressurebased solver approach was undertaken for its better handling of low-speed incompressible flows. In this approach the pressure field is extracted by solving a pressure-correction equation obtained from the SIMPLE (Semi-Implicit Method for Pressure Linked Equations) pressure-velocity coupling scheme. The discretisation of the unstructured tetrahedral mesh used a second-order-upwind scheme in order to obtain sufficiently accurate solutions. For a stable and accurate iterative process, the relaxation factors for momentum and pressure were initially set to 0.5 and 0.2 respectively which were subject to changes depending on the solution which was monitored. In addition, the residual values of the governing equations and the transport equations were all set to converge at 10-5. For the simulation of particle deposition in human airways, a particle tracking method was used to simulate particle transport and evaluate the particle deposition in the airways. All calculations were performed on a Dell P4 3-GHz PC workstation with 2 GB of RAM. A typical run time for the fluid flow and particle tracking was approximately 27 hours.

Blood flows in a stenosed and healthy model are simulated and compared (Figure 3). Newtonian fluid with the density of $1176 \mathrm{~kg} / \mathrm{m} 3$ and viscosity of $0.004 \mathrm{~Pa}$ s. Physiologic flow boundary conditions are imposed. Pulsatile Pressure Waveform is applied at the inlet. Transient simulation was performed for 6 complete cardiac cycles. Physical Time Step: $1 / 100$ of cardiac cycle (0.092 seconds), convergence criterion based on RMS residual was set as $1.0 \times 10^{-4}$. o predict the therapeutic effects before operation, WSS distribution and blood flow are compared between the diseased and Tealth arteries. A hypothesized health model is constructed for this purpose to simulate the situation with stents installed.

Two methods of aerosol medication delivering were simulated and the associated particle size tested was in the range of 3 to 10 micrometers in diameter. The chosen particle size range is typical of those particles from inhalers that can reach the first few generations of airways. Case 1 method requires patient inhale two litres of air in two seconds and then hold the breath for two seconds for aerosol to settle down, however the aerosol only entered in the first $700 \mathrm{ml}$ (milliliters) of air through spacer device. The second method, Case 2, requires patient breath in and out for 5 breaths in two seconds cycle with tidal volume of $400 \mathrm{ml}$; hence 10 seconds of breathing and total volume of air inhaled would be two litres as well. Particles were released at each time step ( 0.005 second $)$ according to the preset particle flow rate which was assumed to be 300 micrograms per second $(\mu \mathrm{g} / \mathrm{s})$. The simulated drug deposition patterns are shown in Figure 4.

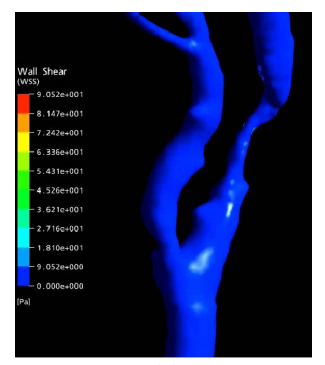

(a)

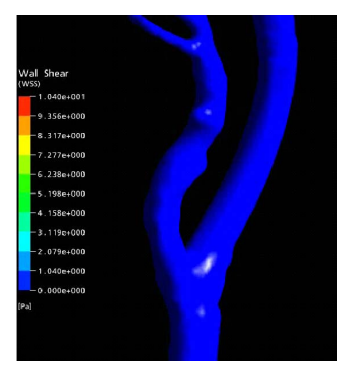

(b) 


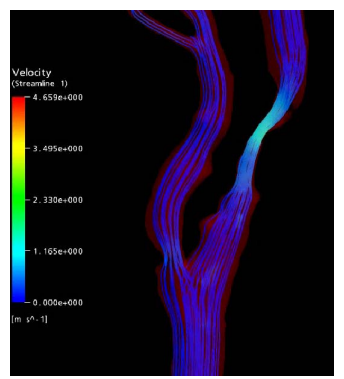

(c)

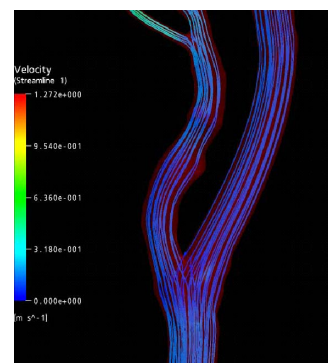

(d)
Figure 3. Comparison of between diseased and health vessels. (a) WSS at diseased vessels. (b)WSS at healthy vessels. (c) Streamline of Blood Flow in diseased vessels (d) Streamline in healthy vessels.

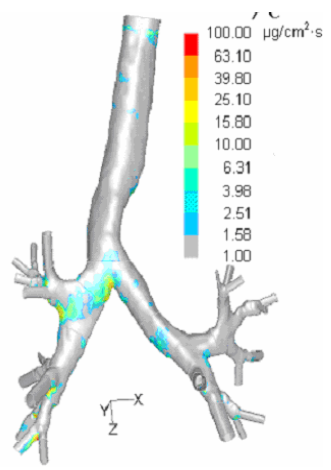

(a)

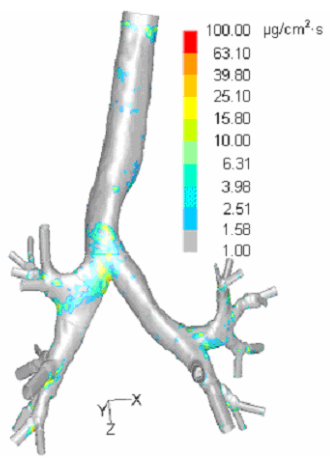

(b)
Figure 4. Drug deposition patterns. (a) Case 1 with particle size of $10 \mu \mathrm{m}\left(\mathrm{St}_{\text {mean } @ \text { inlet }}=0.1544, \mathrm{Re}_{\text {mean@inlet }}=5500\right) .(\mathrm{b})$ Case 2 with particle size of $10 \mu \mathrm{m}\left(\mathrm{St}_{\text {mean @inlet }}=0.07722\right.$, $\operatorname{Re}_{\text {mean } @ i \text { inlet }}=2250$ )

\section{CONCLUSIONS}

This paper presents an improved geometric model construction method to facilitate CFD simulation of blood and air flows. The local constrained watershed algorithm is used in the image processing stage. A new adaptive algorithm is utilized in the second phase to construct the accurate anatomic models. The models generated can be applied by CFD packages directly without any adjustments or modifications. This greatly facilitates downstream research in blood and air flows.

Based on the geometric model of the patient's specific carotid arteries, the hemodynamic parameters will provide doctors with a practical tool that can be used in the diagnosis, therapeutic planning, and postoperative monitoring of patients with arterial diseases. By comparing the blood flow before and after the therapy, this model can be used to assist the physician in making an optimal therapeutic choice. Different breathing patterns are studied in this reserach. The desired or the optimized patterns and parameters are obtainable through automatic comparisons among various distribution patterns by designating the region in which large dose of drugs should be deposited. The best or the most appropriate breathing pattern each individual patient should follow is the one associated with desired drug distribution patterns. Thus, better therapeutic effect could be expected clinically by combining the drug property, patient's specific airway structure and the controllable breathing patterns.

\section{ACKNOWLEDGEMENT}

This research is supported under RMIT Emerging Researcher Grants (2006) and Australian Research Council's linkage Projects funding scheme (LP0561870).

\section{REFERENCES}

[1] L. Jou, C. M. Quick, W. L. Young, and M. T. Lawton, "Computational Approach to Quantifying Hemodynamic Forces in Giant Cerebral Aneurysms", AJNR Am J Neuroradiol, 2003, pp.1804-1810

[2] ]T. Hassan, E. V. Timofeev, T. Saito, H. Shimizu, and M. Ezura, "Computational Replicas: Anatomic Reconstructions of Cerebral Vessels as Volume Numerical Grids at Three-Dimensional Angiography", AJNR Am J Neuroradiol, 2004, pp. 1356-1365.

[3] Y., Zhang and W. H. Finlay," Measurement of the effect of cartilaginous rings on particle deposition in a proximal lung bifurcation model", Aerosol Sci. Tech. 2005, pp.394-399.

[4] M. H. Tawhai, P. Hunter, J. Tschirren, J. Reinhardt, G. McLennan and E. A. Hoffman, "CT-based geometry analysis and finite element models of the human and ovine bronchial tree", J. Appl Physiol , 2004, 97:2310 - 2321.

[5] L. Antiga, B. Ene-Iordache, and A. Remuzzi "Computational geometry for patient-specific reconstruction and meshing of blood bessels from MR and CT angiography", IEEE Transt on Medical Imaging", 2003, Vol. 22(5) pp.674-684.

[6] L. T. Choi, J. Y. Tu, H. F. Li, and F. Thien,. Flow and Particle Deposition Pattern in a Realistic Human Double Bifurcation Airway Model. Inhalation Toxicology. 2007, pp. 117-131.

[7] F. Meyer and S. Beucher, "Morphological Segmentation," J. Visual Comm. And Image Representation, vol. 1, no. 1, pp. 21-46, Sept. 1990.

[8] Richard Beare "A Locally Constrained Watershed Transform", IEEE Transactions On Pattern Analysis And Machine Intelligence, 2006,Vol. 28(7), pp.1063-1074.

[9] M. Eck, and H. Hoppe, "Automatic Reconstruction of B-Spline Surfaces of Arbitrary Topological Type", SIGGRAPH,1996,pp. $325-334$. 